\title{
LOCAL SPECTRUM, LOCAL SPECTRAL RADIUS, AND GROWTH CONDITIONS
}

\author{
HEYBETKULU MUSTAFAYEV
}

\begin{abstract}
Let $X$ be a complex Banach space and $x \in X$. Assume that a bounded linear operator $T$ on $X$ satisfies the condition

$$
\left\|e^{t T} x\right\| \leq C_{x}(1+|t|)^{\alpha} \quad(\alpha \geq 0),
$$

for all $t \in \mathbb{R}$ and for some constant $C_{x}>0$. For the function $f$ from the Beurling algebra $L_{\omega}^{1}(\mathbb{R})$ with the weight $\omega(t)=(1+|t|)^{\alpha}$, we can define an element in $X$, denoted by $x_{f}$, which integrates $e^{t T} x$ with respect to $f$. We present complete description of the elements $x_{f}$ in the case when the local spectrum of $T$ at $x$ consists of one-point. In the case $0 \leq \alpha<1$, some estimates for the norm of $T x$ via local spectral radius of $T$ at $x$ are obtained. Some applications of these results are also given.
\end{abstract}

\section{INTRODUCTION}

Throughout the paper, $X$ will denote a complex Banach space and $B(X)$, the algebra of all bounded linear operators on $X$. As usual, the spectrum and the spectral radius of $T \in B(X)$ will be denoted by $\sigma(T)$ and $r(T)$, respectively. A classical theorem of Gelfand [7] states that if $\sigma(T)=\{1\}$ and $\sup _{n \in \mathbb{Z}}\left\|T^{n}\right\|<\infty$, then $T=I$. A result more general than Gelfand's theorem has been proved by Hille [9]: If $\sigma(T)=\{1\}$ and $\left\|T^{n}\right\|=O\left(|n|^{k}\right)(|n| \rightarrow \infty)$, then $(T-I)^{k+1}=0$. Over the years, these results led to a number of extensions and variations, as shown in the survey article by Zemanek [21.

Continuous version of the Gelfand theorem states that if a quasinilpotent operator $T \in B(X)$ satisfies the condition $\sup _{t \in \mathbb{R}}\left\|e^{t T}\right\|<\infty$, then $T=0$. We give a sketch of the proof:

For an arbitrary $f \in L^{1}(\mathbb{R})$, we can define a bounded linear operator $T_{f}$ on $X$ by

$$
T_{f} x=\int_{\mathbb{R}} f(t) e^{t T} x d t, x \in X .
$$

Then the map $f \mapsto T_{f}$ is a continuous homomorphism. It is easy to check that $I:=\left\{f \in L^{1}(\mathbb{R}): T_{f}=0\right\}$ is a closed ideal of $L^{1}(\mathbb{R})$ whose hull is $\{0\}$. Since $\{0\}$ is a set of synthesis for $L^{1}(\mathbb{R})$, we have $I=\left\{f \in L^{1}(\mathbb{R}): \widehat{f}(0)=0\right\}$, where $\widehat{f}$ is the Fourier transform of $f$. It follows that for a fixed $x \in X$, the functions $t \rightarrow \varphi\left(e^{t T} x\right)$ are constants for every $\varphi \in X^{*}$. Consequently, $\varphi\left(e^{t T} x\right)=\varphi(x)$ for every $\varphi \in X^{*}$ and for all $t \in \mathbb{R}$. So we have $e^{t T} x=x$ for all $x \in X$ and $t \in \mathbb{R}$. This clearly implies that $T=0$.

2010 Mathematics Subject Classification. 47A10, 47A11, 30D20.

Key words and phrases. Operator, (local) spectrum, (local) spectral radius, growth condition, Beurling algebra. 
In this paper, we develop this idea further and prove some results for the local spectrum and local spectral radius for a wider class of operators.

Let $L_{\omega}^{1}(\mathbb{R})$ be the Beurling algebra with the weight $\omega(t)=(1+|t|)^{\alpha}(\alpha \geq 0)$ and let $x \in X$. Assume that $T \in B(X)$ satisfies the condition

$$
\left\|e^{t T} x\right\| \leq C_{x}(1+|t|)^{\alpha},
$$

for all $t \in \mathbb{R}$ and for some constant $C_{x}>0$. For an arbitrary $f \in L_{\omega}^{1}(\mathbb{R})$, we can define an element $x_{f}$ in $X$ by

$$
x_{f}=\int_{\mathbb{R}} f(t) e^{t T} x d t .
$$

We present complete description of the elements $x_{f}$ in the case when the local spectrum of $T$ at $x$ consists of one-point. In the case $0 \leq \alpha<1$, some estimates for the norm of $T x$ via local spectral radius of $T$ at $x$ are given. Some applications of these results are also given.

\section{The Results}

Let us first introduce some basic definitions and results.

For an arbitrary $T \in B(X)$ and $x \in X$, we define $\rho_{T}(x)$ to be the set of all $\lambda \in \mathbb{C}$ for which there exists a neighborhood $U_{\lambda}$ of $\lambda$ with $u(z)$ analytic on $U_{\lambda}$ having values in $X$ such that $(z I-T) u(z)=x$ for all $z \in U_{\lambda}$. This set is open and contains the resolvent set of $T$. By definition, the local spectrum of $T$ at $x \in X$, denoted by $\sigma_{T}(x)$ is the complement of $\rho_{T}(x)$, so it is a compact subset of $\sigma(T)$. This object is most tractable if the operator $T$ has the single-valued extension property (SVEP), i.e. for every open set $U$ in $\mathbb{C}$, the only analytic function $u: U \rightarrow X$ for which the equation $(z I-T) u(z)=0$ holds is the constant function $u \equiv 0$. If $T$ has SVEP, then $\sigma_{T}(x) \neq \emptyset$, whenever $x \in X \backslash\{0\}$ [12, Proposition 1.2.16]. It is easy to see that an operator $T \in B(X)$ having spectrum without interior points has the SVEP.

The local spectral radius of $T \in B(X)$ at $x \in X$ is defined by

$$
r_{T}(x)=\sup \left\{|\lambda|: \lambda \in \sigma_{T}(x)\right\} .
$$

If $T$ has SVEP then

$$
r_{T}(x)=\varlimsup_{n \rightarrow \infty}\left\|T^{n} x\right\|^{\frac{1}{n}}
$$

[12, Proposition 3.3.13].

Recall that a weight function (shortly a weight) $\omega$ is a continuous function on $\mathbb{R}$ such that

$$
\omega(t) \geq 1 \text { and }(t+s) \leq \omega(t) \omega(s), \forall t, s \in \mathbb{R} .
$$

For a weight function $\omega$, by $L_{\omega}^{1}(\mathbb{R})$ we will denote the Banach space of the functions $f \in L^{1}(\mathbb{R})$ with the norm

$$
\|f\|_{1, \omega}=\int_{\mathbb{R}}|f(t)| \omega(t) d t<\infty .
$$

The space $L_{\omega}^{1}(\mathbb{R})$ with convolution product and the norm $\|\cdot\|_{1, \omega}$ is a commutative Banach algebra and is called Beurling algebra. The dual space of $L_{\omega}^{1}(\mathbb{R})$, denoted by $L_{\omega}^{\infty}(\mathbb{R})$, is the space of all measurable functions $g$ on $\mathbb{R}$ such that

$$
\|g\|_{\omega, \infty}:=\operatorname{ess} \sup _{t \in \mathbb{R}} \frac{|g(t)|}{\omega(t)}<\infty .
$$


The duality being implemented by the formula

$$
\langle g, f\rangle=\int_{\mathbb{R}} g(-t) f(t) d t, \forall f \in L_{\omega}^{1}(\mathbb{R}), \forall g \in L_{\omega}^{\infty}(\mathbb{R}) .
$$

Throughout, $[\alpha]$ will denote the integer part of $\alpha \in \mathbb{R}$.

Let $x \in X$ and assume that $T \in B(X)$ satisfies the condition (1.1). For an arbitrary $f \in L_{\omega}^{1}(\mathbb{R})$, we can define an element $x_{f}$ of $X$ by (1.2).

Theorem 2.1. Let $\omega(t)=(1+|t|)^{\alpha}(\alpha \geq 0)$. Assume that $T \in B(X)$ has SVEP and $x \in X$ satisfies the condition $\left\|e^{t T} x\right\| \leq C_{x} \omega(t)$ for all $t \in \mathbb{R}$ and for some constant $C_{x}>0$. Then the following assertions hold:

(a) $\sigma_{T}(x) \subset i \mathbb{R}$.

(b) If $\sigma_{T}(x)=\{i \lambda\}(\lambda \in \mathbb{R})$, then for an arbitrary $f \in L_{\omega}^{1}(\mathbb{R})$,

$$
x_{f}=\widehat{f}(\lambda) x+\frac{\widehat{f}^{\prime}(\lambda)}{1 !}(i T+\lambda I) x+\ldots+\frac{\widehat{f}^{(k)}(\lambda)}{k !}(i T+\lambda I)^{k} x,
$$

where $k=[\alpha]$.

For the proof, we need some preliminary results.

Recall that a weight function $\omega$ is is said to be regular if

$$
\int_{\mathbb{R}} \frac{\log \omega(t)}{1+t^{2}} d t<\infty
$$

For example, $\omega(t)=(1+|t|)^{\alpha}(\alpha \geq 0)$ is a regular weight and it is called polynomial weight. If $\omega$ is a regular weight (in this paper, we will consider regular weights only), then

$$
\lim _{t \rightarrow+\infty} \frac{\log \omega(t)}{t}=\lim _{t \rightarrow+\infty} \frac{\log \omega(-t)}{t}=0 .
$$

Consequently, the maximal ideal space of the algebra $L_{\omega}^{1}(\mathbb{R})$ can be identified with $\mathbb{R}$. The Gelfand transform of $f \in L_{\omega}^{1}(\mathbb{R})$ is just the Fourier transform of $f$. It follows that $L_{\omega}^{1}(\mathbb{R})$ is semisimple. Moreover, the algebra $L_{\omega}^{1}(\mathbb{R})$ is regular (in the Shilov sense). Recall also that $L_{\omega}^{1}(\mathbb{R})$ is Tauberian, that is, the set $\left\{f \in L_{\omega}^{1}(\mathbb{R}): \operatorname{supp} \widehat{f}\right.$ is compact $\}$ is dense in $L_{\omega}^{1}(\mathbb{R})$ (see, [8, Ch.III], [13, Ch.5], and [17, Ch.2]).

We will need also the following well-known fact which is true for every commutative semisimple regular Banach algebra:

If $\left\{I_{\lambda}\right\}_{\lambda \in \Lambda}$ is a collection of the closed ideals of $L_{\omega}^{1}(\mathbb{R})$, then

$$
\operatorname{hull}\left(\bigcap_{\lambda \in \Lambda} I_{\lambda}\right)=\overline{\bigcup_{\lambda \in \Lambda} \operatorname{hull}\left(I_{\lambda}\right)} .
$$

Denote by $M_{\omega}(\mathbb{R})$ the Banach algebra (with respect to convolution product) of all complex regular Borel measures on $\mathbb{R}$ such that

$$
\|\mu\|_{1, \omega}:=\int_{\mathbb{R}} \omega(t) d|\mu|(t)<\infty .
$$

The algebra $L_{\omega}^{1}(\mathbb{R})$ is naturally identifiable with a closed ideal of $M_{\omega}(\mathbb{R})$. By $\widehat{f}$ and $\widehat{\mu}$, we will denote the Fourier and the Fourier-Stieltjes transform of $f \in L_{\omega}^{1}(\mathbb{R})$ and $\mu \in M_{\omega}(\mathbb{R})$, respectively. 
To any closed subset $S$ of $\mathbb{R}$, the following two closed ideals of $L_{\omega}^{1}(\mathbb{R})$ associated:

$$
I_{\omega}(S):=\left\{f \in L_{\omega}^{1}(\mathbb{R}): \widehat{f}(S)=\{0\}\right\}
$$

and

$$
J_{\omega}(S):=\overline{\left\{f \in L_{\omega}^{1}(\mathbb{R}): \operatorname{supp} \widehat{f} \text { is compact and } \operatorname{supp} \widehat{f} \cap S=\emptyset\right\}} .
$$

The ideals $J_{\omega}(S)$ and $I_{\omega}(S)$ are respectively, the smallest and the largest closed ideals in $L_{\omega}^{1}(\mathbb{R})$ with hull $S$. When these two ideals coincide, the set $S$ is said to be a set of synthesis for $L_{\omega}^{1}(\mathbb{R})$ (for instance, see [11, Sect. 8.3]). Notice that

$$
I_{\omega}(\{\infty\})=L_{\omega}^{1}(\mathbb{R}) \text { and } J_{\omega}(\{\infty\})=\overline{\left\{f \in L_{\omega}^{1}(\mathbb{R}): \operatorname{supp} \widehat{f} \text { is compact }\right\}} \text {. }
$$

Since the algebra $L_{\omega}^{1}(\mathbb{R})$ is Tauberian, $I_{\omega}(\{\infty\})=J_{\omega}(\{\infty\})$. Hence, $\{\infty\}$ is a set of synthesis for $L_{\omega}^{1}(\mathbb{R})$.

If $\omega(t)=(1+|t|)^{\alpha}$, then the first $k(=[\alpha])$ derivatives of the Fourier transform of $f \in L_{\omega}^{1}(\mathbb{R})$ exist at each point $\lambda \in \mathbb{R}$ and

$$
J_{\omega}(\{\lambda\})=\left\{f \in L_{\omega}^{1}(\mathbb{R}): \widehat{f}(\lambda)=\widehat{f}^{\prime}(\lambda)=\ldots=\widehat{f}^{(k)}(\lambda)=0\right\}
$$

(for instance, see [8, Ch. IV] and [20]). It follows that if $0 \leq \alpha<1$, then each point (consequently, each finite subset) of $\mathbb{R}$ is a set of synthesis for $L_{\omega}^{1}(\mathbb{R})$.

Let $B_{g}$ be the weak ${ }^{*}$-closed translation invariant subspace of $L_{\omega}^{\infty}(\mathbb{R})$ generated by $g \in L_{\omega}^{\infty}(\mathbb{R})$. The Beurling spectrum $\operatorname{sp}_{B}(g)$ of $g \in L_{\omega}^{\infty}(\mathbb{R})$ is defined by

$$
\operatorname{sp}_{B}(g)=\left\{\lambda \in \mathbb{R}: e^{-i \lambda t} \in B_{g}\right\} .
$$

It is easy to verify that

$$
\operatorname{sp}_{B}(g)=\operatorname{hull}\left(\mathcal{I}_{g}\right)
$$

where

$$
\mathcal{I}_{g}=\left\{f \in L_{\omega}^{1}(\mathbb{R}): f * g=0\right\}
$$

is a closed ideal of $L_{\omega}^{1}(\mathbb{R})$.

The Carleman transform of $g \in L_{\omega}^{\infty}(\mathbb{R})$ is defined as the analytic function $G(z)$ on $\mathbb{C} \backslash i \mathbb{R}$ given by

$$
G(z)=\left\{\begin{array}{l}
\int_{0}^{\infty} e^{-z t} g(t) d t, \operatorname{Re} z>0 ; \\
-\int_{-\infty}^{0} e^{-z t} g(t) d t, \operatorname{Re} z<0 .
\end{array}\right.
$$

We know [10] that $\lambda \in \operatorname{sp}_{B}\{g\}$ if and only if the Carleman transform $G(z)$ of $g$ has no analytic extension to a neighborhood of $i \lambda$.

Let $\omega$ be a weight function, $T \in B(X)$, and let

$$
E_{T}^{\omega}:=\left\{x \in X: \exists C_{x}>0,\left\|e^{t T} x\right\| \leq C_{x} \omega(t), \forall t \in \mathbb{R}\right\} .
$$

Then, $E_{T}^{\omega}$ is a linear (non-closed, in general) subspace of $X$. If $x \in E_{T}^{\omega}$, then for an arbitrary $\mu \in M_{\omega}(\mathbb{R})$, we can define an element $x_{\mu}$ in $X$ by

$$
x_{\mu}=\int_{\mathbb{R}} e^{t T} x d \mu(t) .
$$

Notice that $\mu \mapsto x_{\mu}$ is a bounded linear map from $M_{\omega}(\mathbb{R})$ into $X$ and

$$
\left\|x_{\mu}\right\| \leq C_{x}\|\mu\|_{1, \omega}, \quad \forall \mu \in M_{\omega}(\mathbb{R}) .
$$


Further, from the identity

$$
e^{t T} x_{\mu}=\int_{\mathbb{R}} e^{(t+s) T} x d \mu(s)
$$

we can write

$$
\begin{aligned}
\left\|e^{t T} x_{\mu}\right\| & \leq \int_{\mathbb{R}}\left\|e^{(t+s) T} x\right\| d|\mu|(s) \\
& \leq C_{x} \int_{\mathbb{R}} \omega(t+s) d|\mu|(s) \\
& \leq C_{x} \int_{\mathbb{R}} \omega(t) \omega(s) d|\mu|(s) \\
& =C_{x}\|\mu\|_{1, \omega} \omega(t), \forall t \in \mathbb{R} .
\end{aligned}
$$

This shows that if $x \in E_{T}^{\omega}$, then $x_{\mu} \in E_{T}^{\omega}$ for every $\mu \in M_{\omega}(\mathbb{R})$. In other words, $E_{T}^{\omega}$ is a $M_{\omega}(\mathbb{R})$-module for the map $(\mu, x) \mapsto x_{\mu}$.

It is easy to check that

$$
\left(x_{\mu}\right)_{\nu}=x_{\mu * \nu}, \forall \mu, \nu \in M_{\omega}(\mathbb{R}) .
$$

It follows that if $x \in E_{T}^{\omega}$, then

$$
I_{x}:=\left\{f \in L_{\omega}^{1}(\mathbb{R}): x_{f}=0\right\}
$$

is a closed ideal of $L_{\omega}^{1}(\mathbb{R})$, where

$$
x_{f}=\int_{\mathbb{R}} f(t) e^{t T} x d t .
$$

For a given $x \in E_{T}^{\omega}$, consider the function

$$
u(z):=\left\{\begin{array}{l}
\int_{0}^{\infty} e^{-z t} e^{t T} x d t, \quad \operatorname{Re} z>0 \\
-\int_{-\infty}^{0} e^{-z t} e^{t T} x d t, \quad \operatorname{Re} z<0 .
\end{array}\right.
$$

It follows from $(2.1)$ that $u(z)$ is a function analytic on $\mathbb{C} \backslash i \mathbb{R}$. Let $a:=\operatorname{Re} z>0$. Then, for an arbitrary $s>0$ we can write

$$
(z I-T) \int_{0}^{s} e^{-z t} e^{t T} x d t=-\int_{0}^{s} \frac{d}{d t} e^{t(T-z I)} x d t=x-e^{s(T-z I)} x .
$$

Since

$$
\left\|e^{s(T-z I)} x\right\|=e^{-a s}\left\|e^{s T} x\right\| \leq C_{x} e^{-a s} \omega(s)
$$

and

$$
\lim _{s \rightarrow+\infty} e^{-a s} \omega(s)=0,
$$

we have

$$
(z I-T) u(z)=x, \text { for all } z \in \mathbb{C} \text { with } \operatorname{Re} z>0 .
$$

Similarly,

$$
(z I-T) u(z)=x, \text { for all } z \in \mathbb{C} \text { with } \operatorname{Re} z<0 .
$$

Hence,

$$
(z I-T) u(z)=x, \quad \forall z \in \mathbb{C} \backslash i \mathbb{R} .
$$

This clearly implies that $\sigma_{T}(x) \subset i \mathbb{R}$. 
Thus we have the following:

Proposition 2.2. Let $\omega$ be a regular weight. Assume that $T \in B(X)$ and $x \in X$ satisfy the condition $\left\|e^{t T} x\right\| \leq C_{x} \omega(t)$ for all $t \in \mathbb{R}$ and for some constant $C_{x}>0$. Then $\sigma_{T}(x) \subset i \mathbb{R}$.

Now, assume that $T$ has SVEP. We claim that $\sigma_{T}(x)$ consists of all $\lambda \in i \mathbb{R}$ for which the function $u(z)$ (which is defined by (2.3)) has no analytic extension to a neighborhood of $\lambda$. To see this, let $v(z)$ be the analytic extension of $u(z)$ to a neighborhood $U_{\lambda}$ of $\lambda \in i \mathbb{R}$. It follows from the identity (2.4) that the function

$$
w(z):=(z I-T) v(z)-x
$$

vanishes on $U_{\lambda}^{+}:=\left\{z \in U_{\lambda}: \operatorname{Re} z>0\right\}$ and on $U_{\lambda}^{-}:=\left\{z \in U_{\lambda}: \operatorname{Re} z<0\right\}$. By uniqueness theorem, $w(z)=0$ for all $z \in U_{\lambda}$. So we have

$$
(z I-T) v(z)=x, \forall z \in U_{\lambda} .
$$

This shows that $\lambda \in \rho_{T}(x)$. If $\lambda \in \rho_{T}(x) \cap i \mathbb{R}$, then there exists a neighborhood $U_{\lambda}$ of $\lambda$ with $v(z)$ analytic on $U_{\lambda}$ having values in $X$ such that

$$
(z I-T) v(z)=x, \quad \forall z \in U_{\lambda} .
$$

By (2.4),

$$
(z I-T)(u(z)-v(z))=0, \quad \forall z \in U_{\lambda}^{+}, \forall z \in U_{\lambda}^{-} .
$$

Since $T$ has SVEP, we have

$$
u(z)=v(z), \forall z \in U_{\lambda}^{+}, \forall z \in U_{\lambda}^{-} .
$$

This shows that $u(z)$ can be analytically extended to a neighborhood of $\lambda$.

Let $x \in E_{T}^{\omega}$ be given. For an arbitrary $\varphi \in X^{*}$, define a function $\varphi_{x}$ on $\mathbb{R}$ by

$$
\varphi_{x}(t)=\left\langle\varphi, e^{t T} x\right\rangle \text {. }
$$

Then $\varphi_{x}$ is continuous and

$$
\left|\varphi_{x}(t)\right| \leq C_{x}\|\varphi\| \omega(t), \quad \forall t \in \mathbb{R} .
$$

Consequently, $\varphi_{x} \in L_{\omega}^{\infty}(\mathbb{R})$. Taking into account the identity (2.3), we have

$$
\langle\varphi, u(z)\rangle=\left\{\begin{array}{l}
\int_{0}^{\infty} e^{-z t} \varphi_{x}(t) d t, \quad \operatorname{Re} z>0 ; \\
-\int_{-\infty}^{0} e^{-z t} \varphi_{x}(t) d t, \quad \operatorname{Re} z<0 .
\end{array}\right.
$$

This shows that the function $z \rightarrow\langle\varphi, u(z)\rangle$ is the Carleman transform of $\varphi_{x}$. It follows that

$$
\operatorname{isp}_{B}\left(\varphi_{x}\right) \subseteq \sigma_{T}(x) \quad\left(\forall \varphi \in X^{*}\right)
$$

and hence

$$
\overline{\bigcup_{\varphi \in X^{*}} \operatorname{sp}_{B}\left(\varphi_{x}\right)} \subseteq-i \sigma_{T}(x) .
$$

To show the reverse inclusion, assume that $\lambda_{0} \in \mathbb{R}$ and

$$
\lambda_{0} \notin \overline{\bigcup_{\varphi \in X^{*}} \operatorname{sp}_{B}\left(\varphi_{x}\right)} .
$$

Then there exist a neighborhood $U$ of $\overline{\bigcup_{\varphi \in X^{*}} \operatorname{sp}_{B}\left(\varphi_{x}\right)}$ and $\varepsilon>0$ such that

$$
\left(\lambda_{0}-\varepsilon, \lambda_{0}+\varepsilon\right) \cap \bar{U}=\emptyset .
$$


Since the algebra $L_{\omega}^{1}(\mathbb{R})$ is regular, there exists a function $f \in L_{\omega}^{1}(\mathbb{R})$ such that $\widehat{f}=$ 1 on $\left[\lambda_{0}-\varepsilon / 2, \lambda_{0}+\varepsilon / 2\right]$ and $\widehat{f}=0$ on $\bar{U}$. Notice that $\widehat{f}$ vanishes in a neighborhood of $\operatorname{sp}_{B}\left(\varphi_{x}\right)$ and $\operatorname{supp} \widehat{f} \subseteq\left[\lambda_{0}-\varepsilon, \lambda_{0}+\varepsilon\right]$. Consequently, $f$ belongs to the smallest ideal of $L_{\omega}^{1}(\mathbb{R})$ whose hull is $\operatorname{sp}_{B}\left(\varphi_{x}\right)$. Since

$$
\operatorname{sp}_{B}\left(\varphi_{x}\right)=\operatorname{hull}\left\{f \in L_{\omega}^{1}(\mathbb{R}): f * \varphi_{x}=0\right\},
$$

we have that $\left(\lambda_{0}-\varepsilon / 2, \lambda_{0}+\varepsilon / 2\right) \subset \mathbb{R} \backslash \operatorname{sp}_{B}\left(\varphi_{x}\right)$ for every $\varphi \in X^{*}$. It follows that the function $z \rightarrow\langle\varphi, u(z)\rangle$ can be analytically extended to $i\left(\lambda_{0}-\varepsilon / 2, \lambda_{0}+\varepsilon / 2\right)$ for every $\varphi \in X^{*}$. Hence, $u(z)$ can be analytically extended to $i\left(\lambda_{0}-\varepsilon / 2, \lambda_{0}+\varepsilon / 2\right)$ and therefore $i \lambda_{0} \notin \sigma_{T}(x)$ or $\lambda_{0} \notin-i \sigma_{T}(x)$. Thus we have

$$
\overline{\bigcup_{\varphi \in X^{*}} \operatorname{sp}_{B}\left(\varphi_{x}\right)}=-i \sigma_{T}(x)
$$

Further, it is easy to see that

$$
I_{x}=\bigcap_{\varphi \in X^{*}} \mathcal{I}_{\widetilde{\varphi_{x}}},
$$

where $\widetilde{\varphi_{x}}(t)=\varphi_{x}(-t)$. Taking into account that

$$
\operatorname{sp}_{B}\left(\widetilde{\varphi_{x}}\right)=\left\{-\lambda: \lambda \in \operatorname{sp}_{B}\left(\varphi_{x}\right)\right\},
$$

by $(2.2)$ we can write

$$
\operatorname{hull}\left(I_{x}\right)=\overline{\bigcup_{\varphi \in X^{*}} \operatorname{hull}\left(\mathcal{I}_{\widetilde{\varphi_{x}}}\right)}=\overline{\bigcup_{\varphi \in X^{*}} \operatorname{sp}_{B}\left(\widetilde{\varphi_{x}}\right)}=i \sigma_{T}(x) .
$$

Thus we have the following:

Proposition 2.3. Let $\omega$ be a regular weight. Assume that $T \in B(X)$ has SVEP and $x \in X$ satisfies the condition $\left\|e^{t T} x\right\| \leq C_{x} \omega(t)$ for all $t \in \mathbb{R}$ and for some constant $C_{x}>0$. Then

$$
i \sigma_{T}(x)=\operatorname{hull}\left(I_{x}\right) .
$$

Corollary 2.4. Assume that the hypotheses of Proposition 2.3 are satisfied. The following assertions hold for every $f \in L_{\omega}^{1}(\mathbb{R})$ :

(a) $\sigma_{T}\left(x_{f}\right) \subseteq \sigma_{T}(x)$.

(b) $i \sigma_{T}\left(x_{f}\right) \subseteq \operatorname{supp} \widehat{f}$.

Proof. (a) By Proposition 2.3, it suffices to show that $I_{x} \subseteq I_{x_{f}}$. Indeed, if $g \in I_{x}$, then as $x_{g}=0$ we have

$$
\left(x_{f}\right)_{g}=x_{f * g}=\left(x_{g}\right)_{f}=0 .
$$

This shows that $g \in I_{x_{f}}$.

(b) Assume that the Fourier transform of $g \in L_{\omega}^{1}(\mathbb{R})$ vanishes on supp $\widehat{f}$. Then as $f * g=0$, we have

$$
\left(x_{f}\right)_{g}=x_{f * g}=0
$$

and therefore, $g \in I_{x_{f}}$. Consequently, $\widehat{g}$ vanishes on hull $\left(I_{x_{f}}\right)$. By Proposition 2.3, $\widehat{g}$ vanishes on $i \sigma_{T}\left(x_{f}\right)$.

For $f \in L_{\omega}^{1}(\mathbb{R})$ and $s \in \mathbb{R}$, let $f_{s}(t):=f(t-s)$. Let $e_{n}:=2 n \chi_{[-1 / n, 1 / n]}$ $(n \in \mathbb{N})$, where $\chi_{[-1 / n, 1 / n]}$ is the characteristic function of the interval $[-1 / n, 1 / n]$. 
If $K:=\sup _{t \in[-1,1]} \omega(t)$, then $\left\|e_{n}\right\|_{\omega} \leq K$ for all $n \in \mathbb{N}$. On the other hand, by continuity of the mapping $s \mapsto f_{s}$, we have

$$
\lim _{n \rightarrow \infty}\left\|f * e_{n}-f\right\|_{1, \omega}=0 .
$$

Consequently, $\left\{e_{n}\right\}$ is a bounded approximate identity (b.a.i.) for $L_{\omega}^{1}(\mathbb{R})$. If $x \in$ $E_{T}^{\omega}$, from the identity

$$
x_{e_{n}}-x=\int_{-1 / n}^{1 / n} e_{n}(t)\left(e^{t T} x-x\right) d t
$$

it follows that $x_{e_{n}} \rightarrow x$. Similarly, $x_{f * e_{n}} \rightarrow x_{f}$ for every $f \in L_{\omega}^{1}(\mathbb{R})$.

Proposition 2.5. Let $\omega$ be a regular weight and $x \in X$. Assume that $T \in B(X)$ has $S V E P$ and $\left\|e^{t T} x\right\| \leq C_{x} \omega(t)$ for all $t \in \mathbb{R}$ and for some constant $C_{x}>0$. For an arbitrary $f \in L_{\omega}^{1}(\mathbb{R})$, the following assertions hold:

(a) If $x_{f}=0$, then $\widehat{f}$ vanishes on $i \sigma_{T}(x)$.

(b) If $\widehat{f}$ vanishes in a neighborhood of $i \sigma_{T}(x)$, then $x_{f}=0$.

(c) If $\widehat{f}=1$ in a neighborhood of $i \sigma_{T}(x)$, then $x_{f}=x$.

Proof. (a) By Proposition 2.3, $i \sigma_{T}(x)=\operatorname{hull}\left(I_{x}\right)$ and therefore $I_{x} \subseteq I_{\omega}\left(i \sigma_{T}(x)\right)$. This clearly implies (a).

(b) Let $g \in L_{\omega}^{1}(\mathbb{R})$ be such that supp $\widehat{g}$ is compact. Then $f * g \in J_{\omega}\left(i \sigma_{T}(x)\right)$ and therefore $f * g \in I_{x}$. So we have $x_{f * g}=0$. Since the algebra $L_{\omega}^{1}(\mathbb{R})$ is Tauberian, $x_{f * g}=0$ for all $g \in L_{\omega}^{1}(\mathbb{R})$. It follows that $x_{f * e_{n}}=0$ for all $n$, where $\left\{e_{n}\right\}$ is a b.a.i. for $L_{\omega}^{1}(\mathbb{R})$. As $n \rightarrow \infty$, we have $x_{f}=0$.

(c) Since the Fourier transform of $f * e_{n}-e_{n}$ vanishes in a neighborhood of $i \sigma_{T}(x)$, by (b), $x_{f * e_{n}}=x_{e_{n}}$. As $n \rightarrow \infty$, we have $x_{f}=x$.

As an immediate consequence of Proposition 2.5, we have the following:

Corollary 2.6. Assume that the hypotheses of Proposition 2.5 are satisfied and $f \in L_{\omega}^{1}(\mathbb{R})$. If $i \sigma_{T}(x)$ is a set of synthesis for $L_{\omega}^{1} \mathbb{R}$, then:

(a) $x_{f}=0$ if and only if $\widehat{f}$ vanishes on $i \sigma_{T}(x)$.

(b) If $\widehat{f}=1$ on $i \sigma_{T}(x)$, then $x_{f}=x$.

By $S(\mathbb{R})$ we denote the set of all rapidly decreasing functions on $\mathbb{R}$, i.e. the set of all infinitely differentiable functions $\phi$ on $\mathbb{R}$ such that

$$
\lim _{|t| \rightarrow \infty}\left|t^{n} \phi^{(k)}(t)\right|=0, \forall n, k=0,1,2, \ldots
$$

(here, $n$ can be replaced by any $\alpha \geq 0$ ). It can be seen that if $\omega$ is a polynomial weight, then $S(\mathbb{R}) \subset L_{\omega}^{1}(\mathbb{R})$.

Lemma 2.7. Assume that $T \in B(X)$ and $x \in X$ satisfy the condition

$$
\left\|e^{t T} x\right\| \leq C_{x}(1+|t|)^{\alpha} \quad(\alpha \geq 0),
$$

for all $t \in \mathbb{R}$ and for some constant $C_{x}>0$. Then for an arbitrary $\phi \in S(\mathbb{R})$,

$$
x_{\phi^{(k)}}=(-1)^{k} T^{k} x_{\phi}, \quad \forall k \in \mathbb{N} .
$$

Proof. For an arbitrary $a, b \in \mathbb{R}(a<b)$, we can write

$$
\int_{a}^{b} \phi^{\prime}(t) e^{t T} x d t=\phi(b) e^{b T} x-\phi(a) e^{a T} x-T \int_{a}^{b} \phi(t) e^{t T} x d t .
$$


On the other hand,

$$
\begin{aligned}
\left\|\phi(b) e^{b T} x-\phi(a) e^{a T} x\right\| & \leq C_{x}|\phi(b)|(1+|b|)^{\alpha}+C_{x}|\phi(a)|(1+|a|)^{\alpha} \\
& \leq 2^{\alpha} C_{x}\left(|\phi(b)|+|\phi(b)||b|^{\alpha}+|\phi(a)|+|\phi(a)||a|^{\alpha}\right) .
\end{aligned}
$$

Since $\phi \in S(\mathbb{R})$, it follows that

$$
\lim _{\substack{b \rightarrow+\infty \\ a \rightarrow-\infty}}\left\|\phi(b) e^{b T} x-\phi(a) e^{a T} x\right\|=0 .
$$

Hence $x_{\phi^{\prime}}=-T x_{\phi}$. By induction we obtain our result.

We are now able to prove Theorem 2.1.

Proof of Theorem 2.1. (a) follows from Proposition 2.2. To prove (b), observe that if $\sigma_{T}(x)=\{i \lambda\}(\lambda \in \mathbb{R})$, then $\sigma_{T-i \lambda I}(x)=\{0\}$. Moreover, the operator $T-i \lambda I$ has SVEP and

$$
\left\|e^{t(T-i \lambda I)} x\right\|=\left\|e^{t T} x\right\| \leq C_{x}(1+|t|)^{\alpha} .
$$

Therefore, it suffices to show that if $\sigma_{T}(x)=\{0\}$, then

$$
x_{f}=\widehat{f}(0) x+\frac{\widehat{f}^{\prime}(0)}{1 !}(i T) x+\ldots+\frac{\widehat{f}^{(k)}(0)}{k !}(i T)^{k} x,
$$

for all $f \in L_{\omega}^{1}(\mathbb{R})$. As we have noted above, if $f \in L_{\omega}^{1}(\mathbb{R})$ then the first $k$ derivatives of the Fourier transform of $f$ exist at each point of $s \in \mathbb{R}$ and

$$
J_{\omega}(\{s\})=\left\{f \in L_{\omega}^{1}(\mathbb{R}): \widehat{f}(s)=\widehat{f}^{\prime}(s)=\ldots=\widehat{f}^{(k)}(s)=0\right\},
$$

where $k=[\alpha]$. Recall that $J_{\omega}(\{s\})$ is the smallest closed ideal of $L_{\omega}^{1}(\mathbb{R})$ whose hull is $\{s\}$. By Proposition 2.3, hull $\left(I_{x}\right)=\{0\}$ and therefore, $J_{\omega}(\{0\}) \subseteq I_{x}$. Now, let $\phi \in S(\mathbb{R})$ be such that $\widehat{\phi}=1$ in a neighborhood of 0 . For a given $f \in L_{\omega}^{1}(\mathbb{R})$, consider the function

$$
g:=f-\widehat{f}(0) \phi-\frac{\widehat{f}^{\prime}(0)}{1 !}(-i) \phi^{\prime}-\ldots-\frac{\widehat{f}^{(k)}(0)}{k !}(-i)^{k} \phi^{(k)} .
$$

As

$$
\widehat{\phi^{(k)}}(s)=(i s)^{k} \widehat{\phi}(s)
$$

we have

$$
\widehat{g}(s)=\widehat{f}(s)-\left[\widehat{f}(0)+\frac{\widehat{f}^{\prime}(0)}{1 !} s+\ldots+\frac{\widehat{f}^{(k)}(0)}{k !} s^{k}\right] \widehat{\phi}(s) .
$$

It can be seen that the first $k$ derivatives of the function $\widehat{g}$ at 0 are zero and therefore, $g \in J_{\omega}(\{0\})$. Consequently, $g \in I_{x}$ and so $x_{g}=0$. On the other hand, by Lemma 2.7 ,

which implies

$$
x_{\phi^{(k)}}=(-1)^{k} T^{k} x
$$

$$
x_{g}=x_{f}-\widehat{f}(0) x-\frac{\widehat{f}^{\prime}(0)}{1 !}(i T) x-\ldots-\frac{\widehat{f}^{(k)}(0)}{k !}(i T)^{k} x .
$$

So we have

$$
x_{f}=\widehat{f}(0) x+\frac{\widehat{f}^{\prime}(0)}{1 !}(i T) x+\ldots+\frac{\widehat{f}^{(k)}(0)}{k !}(i T)^{k} x .
$$


Assume that $T \in B(X)$ has SVEP, $(T-\lambda I)^{k} x=0$, and $(T-\lambda I)^{k-1} x \neq 0$ for some $x \in X, \lambda \in \mathbb{C}$, and $k \in \mathbb{N}$. We claim that $\sigma_{T}(x)=\{\lambda\}$. Indeed, it is easy to check that if

$$
u(z):=\frac{x}{z-\lambda}+\frac{(T-\lambda I) x}{(z-\lambda)^{2}}+\ldots+\frac{(T-\lambda I)^{k-1} x}{(z-\lambda)^{k}},
$$

then $(z I-T) u(z)=x$ for all $z \in \mathbb{C} \backslash\{\lambda\}$. This shows that $\sigma_{T}(x) \subseteq\{\lambda\}$. Since $T$ has SVEP and $\sigma_{T}(x) \neq \emptyset$, we have $\sigma_{T}(x)=\{\lambda\}$.

As a consequence of Theorem 2.1, we have the following:

Corollary 2.8. Assume that the hypotheses of Theorem 2.1 are satisfied. If $\sigma_{T}(x)=$ $\{i \lambda\}(\lambda \in \mathbb{R})$, then $(T-i \lambda I)^{[\alpha]+1} x=0$.

Proof. As in the proof of Theorem 2.1, we may assume that $\sigma_{T}(x)=\{0\}$. Let us show that $T^{k+1} x=0$, where $k=[\alpha]$. Let $\phi \in S(\mathbb{R})$ be such that $\widehat{\phi}=1$ in a neighborhood of 0 and $f:=\phi^{(k+1)}$. Since

$$
\widehat{f}(0)=\widehat{f}^{\prime}(0)=\ldots=\widehat{f}^{(k)}(0)=0,
$$

by Theorem 2.1 ,

$$
0=x_{f}=(-1)^{k+1} T^{k+1} x .
$$

Corollary 2.9. Assume that the hypotheses of Theorem 2.1 are satisfied and $0 \leq$ $\alpha<1$. If $\sigma_{T}(x)=\left\{i \lambda_{1}, \ldots, i \lambda_{N}\right\}$, where $\lambda_{1}, \ldots, \lambda_{N}$ are pairwice distinct real numbers, then there exist $x_{1}, \ldots, x_{N}$ in $X$ such that $x=x_{1}+\ldots+x_{N}$ and $T x_{k}=i \lambda_{k} x_{k}$ $(k=1, \ldots, N)$.

Proof. Let $f \in L_{\omega}^{1}(\mathbb{R})$ be such that

$$
\widehat{f}\left(-\lambda_{1}\right)=\ldots=\widehat{f}\left(-\lambda_{N}\right)=1 .
$$

Since $\left\{-\lambda_{1}, \ldots,-\lambda_{N}\right\}$ is a set of synthesis for $L_{\omega}^{1}(\mathbb{R})$, by Corollary $2.6, x_{f}=x$. Now, assume that the functions $f_{1}, \ldots, f_{N}$ in $L_{\omega}^{1}(\mathbb{R})$ satisfy the conditions

$$
\widehat{f_{m}}\left(-\lambda_{n}\right)=\delta_{m n} \quad(m, n=1, \ldots, N)
$$

where $\delta_{m n}$ is the Kronecker symbol. We put $x_{k}=x_{f_{k}}$. Since the Fourier transform of the function $f-f_{1}-\ldots-f_{N}$ vanishes on $\left\{-\lambda_{1}, \ldots,-\lambda_{N}\right\}$, by Corollary 2.6,

$$
x=x_{f}=x_{1}+\ldots+x_{N} .
$$

On the other hand, by Corollary $2.4, \sigma_{T}\left(x_{k}\right) \subseteq\left\{i \lambda_{k}\right\}$. Since $T$ has SVEP, $\sigma_{T}\left(x_{k}\right)=$ $\left\{i \lambda_{k}\right\}$. By Corollary 2.8, $T x_{k}=i \lambda_{k} x_{k}(k=1, \ldots, N)$.

Example 2.10. The condition $\left\|e^{t T} x\right\| \leq C_{x}(1+t)^{\alpha}$ for all $t \geq 0$ is not sufficient in Corollary 2.8. To see this, let $S$ be a contraction on a Hilbert space $H$ such that $\sigma(S)=\{1\}$ and $S \neq I$ (if $S$ is a $C_{0}$-contraction on $H$ with minimal function $\exp \frac{z+1}{z-1}$, then $\sigma(S)=\{1\}$ and $S \neq I$ [16. Ch.III]). If $T:=S-I$, then $T \neq 0$, $\sigma(T)=0$, and $\left\|e^{t T} x\right\| \leq\|x\|$ for all $x \in H$ and $t \geq 0$. Let $x_{0} \in H$ be such that $T x_{0} \neq 0$. Since $x_{0} \neq 0$ and $T$ has SVEP, $\sigma_{T}\left(x_{0}\right) \neq \emptyset$. On the other hand, as $\sigma_{T}\left(x_{0}\right) \subseteq \sigma(T)=\{0\}$, we have $\sigma_{T}\left(x_{0}\right)=\{0\}$. 
Assume that the condition (1.1) is satisfied for all $x \in X$. Then, by the uniform boundedness principle there exists a constant $C_{T}>0$ such that

$$
\left\|e^{t T}\right\| \leq C_{T}(1+|t|)^{\alpha}, \quad \forall t \in \mathbb{R} .
$$

Let $T$ be the backward shift operator on the Hardy space $H^{2}$ defined by

$$
(T f)(z)=\frac{f(z)-f(0)}{z} .
$$

From this it is readily verified that

$$
\left\|e^{t T} z^{k}\right\|_{2} \leq C_{k}(1+|t|)^{k}, \quad \forall k \in \mathbb{N} \cup\{0\}
$$

It follows that the operator $T$ does not satisfy the condition (2.5).

We recall that according to [12, Definition 1.1.1], an operator $T \in B(X)$ is decomposable if for every cover of the complex plane by a pair $\{U, W\}$ of open sets, there exist a pair of closed $T$-invariant subspaces $E$ and $F$ of $X$ such that $E+F=X, \sigma\left(\left.T\right|_{E}\right) \subset U$, and $\sigma\left(\left.T\right|_{F}\right) \subset W$.

Every decomposable operator $T$ on a Banach space $X$ has SVEP [12, Ch.1]. Moreover, $\lim _{n \rightarrow \infty}\left\|T^{n} x\right\|^{\frac{1}{n}}$ exists for all $x \in X$ [12, Proposition 3.3.17].

For an arbitrary $k \in\{0,1, \ldots, \infty\}$, let $C^{k}(\Omega)$ be the algebra of all $k$-times continuously differentiable complex-valued functions defined on an open subset $\Omega$ of the complex plane. An operator $A \in B(X)$ is said to be $C^{k}(\Omega)$-scalar operator if there exists a continuous algebra homomorphism $\Phi: C^{k}(\Omega) \rightarrow B(X)$ for which $\Phi(\mathbf{1})=I$ and $\Phi(\mathbf{z})=A$ [5, 12]. In the case $\Omega=\mathbb{C}$ and $k=\infty$, the operator $A$ is said to be generalized scalar (in this case, $\Phi$ is continuous with respect to the natural Fréchet algebra topology on $\left.C^{\infty}(\mathbb{C})\right)$. It is known [12, Theorem 1.4.10] that every $C^{k}(\Omega)$-scalar operator is decomposable.

Every generalized scalar operator $A$ has a decomposition $A=T+i S$ with $T, S$ commuting generalized scalar operators with real spectra [5, Lemma 4.6.1]. Recall also [12, Theorem 1.5.19] that an operator $T$ with real spectrum is generalized scalar if and only if there exist constants $C_{T}>0$ and $\alpha \geq 0$ such that

$$
\left\|e^{i t T}\right\| \leq C_{T}\left(1+|t|^{\alpha}\right), \forall t \in \mathbb{R} .
$$

An operator $T \in B(X)$ is said to be Hermitian if $\left\|e^{i t T}\right\|=1$ for all $t \in \mathbb{R}$. A Hermitian operator is $C^{1}(\mathbb{R})$-scalar and therefore it is decomposable [2]. A basis result for Hermitian operators is that $\|T\|=r(T)$ [3, p.57].

We have the following:

Theorem 2.11. Assume that $T \in B(X)$ has $S V E P$ and $x \in X$ satisfies the condition

$$
\left\|e^{t T} x\right\| \leq C_{x}\left(1+|t|^{\alpha}\right) \quad(0 \leq \alpha<1),
$$

for all $t \in \mathbb{R}$ and for some constant $C_{x}>0$. Then we have

$$
\|T x\| \leq C_{x}\left[r_{T}(x)+C(\alpha) r_{T}(x)^{1-\alpha}\right]
$$

where

$$
C(\alpha)=\left(\frac{2}{\pi}\right)^{2-\alpha} \sum_{k \in \mathbb{Z}} \frac{1}{|2 k+1|^{2-\alpha}} \quad(C(0)=1) .
$$

The following lemma is needed for the proof of the theorem. 
HEYBETKULU MUSTAFAYEV

Lemma 2.12. Let $\mu \in M_{\omega}(\mathbb{R})$, where $\omega(t)=(1+|t|)^{\alpha}(\alpha \geq 0)$. Assume that $T \in B(X)$ and $x \in X$ satisfy the following conditions:

(i) $T$ has $S V E P$;

(ii) $\left\|e^{t T} x\right\| \leq C_{x} \omega(t)$ for all $t \in \mathbb{R}$ and for some constant $C_{x}>0$.

If $\widehat{\mu}(\lambda)=\lambda$ in a neighborhood of $i \sigma_{T}(x)$, then

$$
x_{\mu}=i T x .
$$

Proof. Let $g \in S(\mathbb{R})$ be such that $\widehat{g}=1$ in a neighborhood of $i \sigma_{T}(x)$. By Proposition $2.5, x_{g}=x$. On the other hand, by Lemma 2.7 ,

$$
x_{g^{\prime}}=-T x \text {. }
$$

Since

$$
\widehat{g^{\prime}}(\lambda)=i \lambda \widehat{g}(\lambda),
$$

the Fourier transform of the function $-i g^{\prime}-\mu * g$ vanishes in a neighborhood of $i \sigma_{T}(x)$. By Proposition 2.5,

$$
-i x_{g^{\prime}}=x_{\mu * g}=\left(x_{g}\right)_{\mu}=x_{\mu} .
$$

Hence $x_{\mu}=i T x$.

Note that in the preceding lemma the weight function $\omega(t)=(1+|t|)^{\alpha}(\alpha \geq 0)$ can be replaced by the weight $\omega(t)=1+|t|^{\alpha}(0 \leq \alpha<1)$.

Proof of Theorem 2.11. We basically follow the proof in 14, Lemma 3.4]. Let $a>0$ be such that $i \sigma_{T}(x) \subset(-a, a)$. Consider the function $f$ on $\mathbb{R}$ defined by $f(\lambda)=\lambda$ for $-a \leq \lambda \leq a$ and $f(\lambda)=2 a-\lambda$ for $a \leq \lambda \leq 3 a$. We extend this function periodically to the real line by putting $f(\lambda+4 a)=f(\lambda)(\lambda \in \mathbb{R})$. The Fourier coefficients of $f$ are given by the equalities

$$
c_{n}(f)=\frac{1}{4 a} \int_{-a}^{3 a} \exp \left(-i \frac{n \pi}{2 a} \lambda\right) f(\lambda) d \lambda .
$$

A few lines of computation show that

$$
c_{2 k}(f)=0 \text { and } c_{2 k+1}(f)=\frac{1}{i} \frac{4 a}{\pi^{2}}(-1)^{k} \frac{1}{(2 k+1)^{2}} \quad(k \in \mathbb{Z}) .
$$

Let $\mu$ be a discrete measure on $\mathbb{R}$ concentrated at the points

$$
\lambda_{k}:=-\frac{1}{a}(2 k+1) \frac{\pi}{2}
$$

with the corresponding weights

$$
c_{k}:=\frac{1}{i} \frac{4 a}{\pi^{2}}(-1)^{k} \frac{1}{(2 k+1)^{2}} \quad(k \in \mathbb{Z}) .
$$

Since

$$
\sum_{k \in \mathbb{Z}}\left|c_{k}\right|<\infty
$$

by the uniqueness theorem,

$$
\widehat{\mu}(\lambda)=f(\lambda)=\frac{1}{i} \frac{4 a}{\pi^{2}} \sum_{k \in \mathbb{Z}}(-1)^{k} \frac{1}{(2 k+1)^{2}} \exp \left[i \frac{1}{a}(2 k+1) \frac{\pi}{2} \lambda\right] .
$$


Now if $\omega(t):=1+|t|^{\alpha}(0 \leq \alpha<1)$, then as

$$
\sum_{k \in \mathbb{Z}} \frac{1}{(2 k+1)^{2}}=\frac{\pi^{2}}{4}
$$

we can write

$$
\begin{aligned}
\|\mu\|_{1, \omega} & =\int_{\mathbb{R}}\left(1+|t|^{\alpha}\right) d|\mu|(t) \\
& =\sum_{k \in \mathbb{Z}}\left|c_{k}\right|\left(1+\left|\lambda_{k}\right|^{\alpha}\right) \\
& =\frac{4 a}{\pi^{2}} \sum_{k \in \mathbb{Z}} \frac{\left[1+\frac{1}{a^{\alpha}}|2 k+1|^{\alpha}\left(\frac{\pi}{2}\right)^{\alpha}\right]}{(2 k+1)^{2}} \\
& =a+C(\alpha) a^{1-\alpha},
\end{aligned}
$$

where

$$
C(\alpha)=\left(\frac{2}{\pi}\right)^{2-\alpha} \sum_{k \in \mathbb{Z}} \frac{1}{|2 k+1|^{2-\alpha}} .
$$

Since $\widehat{\mu}(\lambda)=\lambda$ in a neighborhood of $i \sigma_{T}(x)$, by Lemma 2.12, $x_{\mu}=i T x$. Consequently, we get

$$
\|T x\|=\left\|x_{\mu}\right\| \leq C_{x}\|\mu\|_{1, \omega}=C_{x}\left[a+C(\alpha) a^{1-\alpha}\right] .
$$

Since $a$ is an arbitrary positive number greater than $r_{T}(x)$, we have

$$
\|T x\| \leq C_{x}\left[r_{T}(x)+C(\alpha) r_{T}(x)^{1-\alpha}\right] .
$$

An obvious corollary of Theorem 2.11 is the next result.

Corollary 2.13. If the operator $T \in B(X)$ satisfies the condition (2.6) with $0 \leq$ $\alpha<1$, then for every $x \in X$,

$$
\|T x\| \leq C_{T}\left[r_{T}(x)+C(\alpha) r_{T}(x)^{1-\alpha}\right]\|x\| .
$$

In particular, we have

$$
\|T\| \leq C_{T}\left[r(T)+C(\alpha) r(T)^{1-\alpha}\right] .
$$

where $C(\alpha)$ is defined by $(2.7)$.

An operator $A \in B(X)$ is called normal if $A=T+i S$ with $T, S$ are commuting Hermitian operators on $X$. A normal operator on a Banach space is $C^{2}(\mathbb{C})$-scalar and therefore it is decomposable [2. If $A$ is a normal operator on a Banach space, then $\|A\| \leq 2 r(A)$ (see, Lemma 2.15). In [2, Corollary 3], it was proved that if $A=T+i S$ is a normal operator on a Banach space $X$ and $r_{A}(x)=0$ for some $x \in X$, then $T x=S x=0$. More general result was obtained in [4, Corollary 4]:

$$
\max \{\|T x\|,\|S x\|\} \leq r_{A}(x)\|x\|, \quad \forall x \in X .
$$

We have the following: 
Theorem 2.14. Let $T$ and $S$ are two commuting operators in $B(X)$ satisfying the condition (2.6) with constants $\left\{C_{T}, \alpha\right\}$ and $\left\{C_{S}, \alpha\right\}$, respectively. If $0 \leq \alpha<1$, then for every $x \in X$,

$$
\max \{\|T x\|,\|S x\|\} \leq \max \left\{C_{T}, C_{S}\right\}\left[r_{T+i S}(x)+C(\alpha) r_{T+i S}(x)^{1-\alpha}\right]\|x\|,
$$

where $C(\alpha)$ is defined by $(2.7)$.

For the proof, we need some preliminary results.

Lemma 2.15. If $T$ and $S$ are two commuting operators in $B(X)$ with real spectra, then

$$
\max \{r(T), r(S)\} \leq r(T+i S) .
$$

Proof. Let $\mathcal{A}$ be the maximal commutative subalgebra of $B(X)$ containing $T$ and $S$. Clearly, $\mathcal{A}$ is unital. Denote by $\sigma_{\mathcal{A}}(A)$ the spectrum of $A \in \mathcal{A}$ with respect to the algebra $\mathcal{A}$. Let $\Sigma_{\mathcal{A}}$ be the maximal ideal space of $\mathcal{A}$. Since $\mathcal{A}$ is a full subalgebra of $B(X)$, we have

$$
\begin{aligned}
\sigma(T+i S) & =\sigma_{\mathcal{A}}(T+i S) \\
& =\left\{\phi(T+i S): \phi \in \Sigma_{\mathcal{A}}\right\} \\
& =\left\{\phi(T)+i \phi(S): \phi \in \Sigma_{\mathcal{A}}\right\}
\end{aligned}
$$

and therefore,

$$
r(T+i S)=\sup _{\phi \in \Sigma_{\mathcal{A}}}|\phi(T)+i \phi(S)|
$$

Similarly, as

$$
\sigma(T)=\left\{\phi(T): \phi \in \Sigma_{\mathcal{A}}\right\} \text { and } \sigma(S)=\left\{\phi(S): \phi \in \Sigma_{\mathcal{A}}\right\},
$$

we have

$$
r(T)=\sup _{\phi \in \Sigma_{\mathcal{A}}}|\phi(T)| \text { and } r(S)=\sup _{\phi \in \Sigma_{\mathcal{A}}}|\phi(S)| .
$$

Since $\phi(T)$ and $\phi(S)$ are reals, we obtain that

$$
r(T) \leq r(T+i S) \text { and } r(S) \leq r(T+i S) .
$$

For a closed subset $F$ of $\mathbb{C}$, the local spectral subspace $X_{T}(F)$ of $T \in B(X)$ is defined by

$$
X_{T}(F)=\left\{x \in X: \sigma_{T}(x) \subseteq F\right\} .
$$

An operator $T \in B(X)$ has Dunford's property $(C)$ if $X_{T}(F)$ is closed for every closed set $F \subset \mathbb{C}$. Every decomposable operator has Dunford's property $(C)$ [12, Theorem 1.2.23]. If $T$ has Dunford's property $(C)$, then $\sigma(T)=\sigma_{T}(x)$ holds for every cyclic vector $x$ for $T$ [12, p.238].

Lemma 2.16. Let $T$ and $S$ are two commuting operators in $B(X)$ satisfying the condition (2.6) with constants $\left\{C_{T}, \alpha\right\}$ and $\left\{C_{S}, \beta\right\}$, respectively. Then, for every $x \in X$ one has

$$
\max \left\{r_{T}(x), r_{S}(x)\right\} \leq r_{T+i S}(x) .
$$


Proof. Note that the operator $T+i S$ is $C^{k}(\mathbb{C})$-scalar with $k>\alpha+\beta+1$ [1, Satz 3.3 and Lemma 3.5]. Consequently, $T+i S$ is decomposable and therefore it has Dunford's property $(C)$. For a given $x \in X$, let $Y$ denote the closed linear span of

$$
\left\{T^{n} S^{m} x: n, m \in \mathbb{N} \cup\{0\}\right\} .
$$

Then the space $Y$ is invariant under $T+i S$. Consequently, the operator $\left.T\right|_{Y}+\left.i S\right|_{Y}$ also has Dunford's property $(C)$ [12, Proposition 1.2.21]. Since $x$ is a cyclic vector of $\left.T\right|_{Y}+\left.i S\right|_{Y}$, we have

$$
r\left(\left.T\right|_{Y}+\left.i S\right|_{Y}\right)=r_{T+i S}(x) .
$$

Observe that the operators $\left.T\right|_{Y}$ and $\left.S\right|_{Y}$ also satisfy the condition (2.6) with constants $\left(C_{T}, \alpha\right)$ and $\left(C_{S}, \beta\right)$, respectively. Therefore, the spectra of the operators $\left.T\right|_{Y}$ and $\left.S\right|_{Y}$ are reals. By Lemma 2.15,

$$
\max \left\{r\left(\left.T\right|_{Y}\right), r\left(\left.S\right|_{Y}\right)\right\} \leq r\left(\left.T\right|_{Y}+\left.i S\right|_{Y}\right)=r_{T+i S}(x) .
$$

Since $\sigma_{T}(x) \subseteq \sigma_{\left.T\right|_{Y}}(x)$, we get

$$
r_{T}(x) \leq r_{\left.T\right|_{Y}}(x) \leq r\left(\left.T\right|_{Y}\right) .
$$

Similarly, $r_{S}(x) \leq r\left(\left.S\right|_{Y}\right)$. So we have

$$
\max \left\{r_{T}(x), r_{S}(x)\right\} \leq \max \left\{r\left(\left.T\right|_{Y}\right), r\left(\left.S\right|_{Y}\right)\right\} \leq r_{T+i S}(x) .
$$

Now we can prove Theorem 2.14.

Proof of Theorem 2.14. For an arbitrary $x \in X$, by Corollary 2.13 and Lemma 2.16 , we can write

$$
\begin{aligned}
\|T x\| & \leq C_{T}\left[r_{T}(x)+C(\alpha) r_{T}(x)^{1-\alpha}\right]\|x\| \\
& \leq C_{T}\left[r_{T+i S}(x)+C(\alpha) r_{T+i S}(x)^{1-\alpha}\right]\|x\| .
\end{aligned}
$$

Similarly,

$$
\|S x\| \leq C_{S}\left[r_{T+i S}(x)+C(\alpha) r_{T+i S}(x)^{1-\alpha}\right]\|x\| .
$$

So we have

$$
\max \{\|T x\|,\|S x\|\} \leq \max \left\{C_{T}, C_{S}\right\}\left[r_{T+i S}(x)+C(\alpha) r_{T+i S}(x)^{1-\alpha}\right]\|x\| .
$$

\section{Applications}

For an invertible operator $A$ on a Hilbert space $H$, Deddens [6] introduced the set

$$
\mathcal{B}_{A}:=\left\{T \in B(H): \sup _{n \in \mathbb{N}}\left\|A^{n} T A^{-n}\right\|<\infty\right\} .
$$

Notice that $\mathcal{B}_{A}$ is a normed (not necessarily closed) algebra with identity and contains the commutant $\{A\}^{\prime}$ of $A$. In the same paper, Deddens shows that if $A \geq 0$, then $\mathcal{B}_{A}$ coincides with the nest algebra associated with the nest of spectral subspaces of $A$. This gives a new and convenient characterization of nest algebras. In [6], Deddens conjectured that the equality $\mathcal{B}_{A}=\{A\}^{\prime}$ holds if the spectrum of $A$ is reduced to $\{1\}$. In [18, Roth gave a negative answer to Deddens conjecture. Williams [19] proved that if $A \in B(X)$ is a quasinilpotent and $T \in B(X)$ satisfies 
the condition $\sup _{t \in \mathbb{R}}\left\|e^{t A} T e^{-t A}\right\|<\infty$, then $A T=T A$ (for related results see also [15]).

For a fixed $A \in B(X)$ and $\alpha \geq 0$, we consider the class $\mathcal{D}_{A}^{\alpha}(\mathbb{R})$ of all operators $T \in B(X)$ for which there exists a constant $C_{T}>0$ such that

$$
\left\|e^{t A} T e^{-t A}\right\| \leq C_{T}(1+|t|)^{\alpha}, \quad \forall t \in \mathbb{R} .
$$

Notice that $\mathcal{D}_{A}^{\alpha}(\mathbb{R})$ is a linear (not necessarily closed) subspace of $B(X)$ and contains the commutant of $A$.

As an applications of the results of the preceding section, here we give complete characterization of the class $\mathcal{D}_{A}^{\alpha}(\mathbb{R})$ in the case when the spectrum of $A$ consists of one-point.

Let $A \in B(X)$ and $\Delta_{A}$ be the inner derivation on $B(X)$;

$$
\Delta_{A}: T \mapsto A T-T A, T \in B(X) .
$$

We have

$$
\Delta_{A}^{n}(T)=\sum_{k=0}^{n}(-1)^{k}\left(\begin{array}{l}
n \\
k
\end{array}\right) A^{n-k} T A^{k} \quad(n \in \mathbb{N}) .
$$

Theorem 3.1. For every operator $A \in B(X)$ with real spectrum we have

$$
\mathcal{D}_{A}^{\alpha}(\mathbb{R})=\operatorname{ker} \Delta_{A}^{[\alpha]+1} .
$$

In particular if $0 \leq \alpha<1$, then $\mathcal{D}_{A}^{\alpha}(\mathbb{R})=\{A\}^{\prime}$.

Proof. If $T \in \mathcal{D}_{A}^{\alpha}(\mathbb{R})$, then as

$$
e^{t A} T e^{-t A}=e^{t \Delta_{A}}(T),
$$

we have

$$
\left\|e^{t \Delta_{A}}(T)\right\| \leq C_{T}(1+|t|)^{\alpha}, \quad \forall t \in \mathbb{R}
$$

By Proposition 2.2, $\sigma_{\Delta_{A}}(T) \subset i \mathbb{R}$. Further, since $\sigma(A) \subset \mathbb{R}$ and

$$
\sigma\left(\Delta_{A}\right)=\{\lambda-\mu: \lambda, \mu \in \sigma(A)\}
$$

[12, Theorem 3.5.1], we have $\sigma\left(\Delta_{A}\right) \subset \mathbb{R}$. Therefore $\Delta_{A}$ has SVEP. On the other hand, as

$$
\sigma_{\Delta_{A}}(T) \subseteq \sigma\left(\Delta_{A}\right) \subset \mathbb{R}
$$

we get

$$
\sigma_{\Delta_{A}}(T) \subseteq \mathbb{R} \cap i \mathbb{R}=\{0\}
$$

Since $\Delta_{A}$ has SVEP, $\sigma_{\Delta_{A}}(T) \neq \emptyset$ and so $\sigma_{\Delta_{A}}(T)=\{0\}$. Applying Corollary 2.8 to the operator $\Delta_{A}$ on the space $B(X)$, we obtain that

$$
\Delta_{A}^{[\alpha]+1}(T)=0 .
$$

For the reverse inclusion, let $T \in B(X)$ and assume that there exists $n \in \mathbb{N}$ such that $\Delta_{A}^{n+1}(T)=0$ and $\Delta_{A}^{n}(T) \neq 0$. Then

$$
\begin{aligned}
\left\|e^{t A} T e^{-t A}\right\| & =\left\|e^{t \Delta_{A}}(T)\right\| \\
& =\left\|I+\frac{t \Delta_{A}(T)}{1 !}+\ldots+\frac{t^{n} \Delta_{A}^{n}(T)}{n !}\right\| \\
& =O(1+|t|)^{n} \quad(|t| \rightarrow \infty) .
\end{aligned}
$$

This shows that $T \in \mathcal{D}_{A}^{n}(\mathbb{R})$.

The following corollary generalizes the Williams result mentioned above. 
Corollary 3.2. For every operator $A \in B(X)$ with one-point spectrum, we have

$$
\mathcal{D}_{A}^{\alpha}(\mathbb{R})=\operatorname{ker} \Delta_{A}^{[\alpha]+1} .
$$

In particular if $0 \leq \alpha<1$, then $\mathcal{D}_{A}^{\alpha}(\mathbb{R})=\{A\}^{\prime}$.

Proof. Assume that $\sigma(A)=\{\lambda\}$. If $T \in \mathcal{D}_{A}^{\alpha}(\mathbb{R})$ then $T \in \mathcal{D}_{B}^{\alpha}(\mathbb{R})$, where $B=$ $A-\lambda I$. Since $\sigma(B)=\{0\}$ and $\Delta_{B}^{n}(T)=\Delta_{A}^{n}(T)$ for all $n \in \mathbb{N}$, by Theorem 3.1 we obtain our result.

Example 3.3. If $\alpha \geq 1$, then $\mathcal{D}_{A}^{\alpha}(\mathbb{R}) \neq\{A\}^{\prime}$, in general. To see this, let $A=$ $\left(\begin{array}{ll}0 & 0 \\ 1 & 0\end{array}\right)$ and $T=\left(\begin{array}{ll}1 & 0 \\ 0 & 0\end{array}\right)$ be two $2 \times 2$ matrices on 2 -dimensional Hilbert space. As $A^{2}=0$, we have $\sigma(A)=\{0\}$ and

$$
e^{t A} T e^{-t A}=(I+t A) T(I-t A)=\left(\begin{array}{ll}
1 & 0 \\
t & 0
\end{array}\right), \forall t \in \mathbb{R} .
$$

Since

$$
\left\|e^{t A} T e^{-t A}\right\|=\left(1+|t|^{2}\right)^{\frac{1}{2}}
$$

we have $T \in \mathcal{D}_{A}^{1}(\mathbb{R})$, but $A T \neq T A$.

Example 3.4. The condition $\left\|e^{t A} T e^{-t A}\right\| \leq C_{T}(1+t)^{\alpha}$ for all $t \geq 0$ is not sufficient in Corollary 3.2. To see this, let $S$ be a contraction on a Hilbert space $H$ such that $\sigma(S)=\{1\}$ and $S \neq I$ (see, Example 2.10). If $Q:=S-I$, then $\sigma(Q)=\{0\}$ and $\left\|e^{t Q}\right\| \leq 1$ for all $t \geq 0$. Define the operator $A$ on $H \oplus H$ by $A=\left(\begin{array}{cc}-Q & 0 \\ 0 & 0\end{array}\right)$. Since $e^{t A}=\left(\begin{array}{cc}e^{-t Q} & 0 \\ 0 & I\end{array}\right)$, for $T=\left(\begin{array}{cc}0 & 0 \\ I & 0\end{array}\right)$, we have

$$
e^{t A} T e^{-t A}=\left(\begin{array}{cc}
0 & 0 \\
e^{t Q} & 0
\end{array}\right) \text {. }
$$

It follows that

$$
\left\|e^{t A} T e^{-t A}\right\|=\left\|e^{t Q}\right\| \leq 1(\forall t \geq 0)
$$

but $A T \neq T A$.

Next, we give some results related to the decomposability.

Proposition 3.5. Assume that $A \in B(X)$ is decomposable and $T \in B(X)$ satisfies the condition

$$
\left\|e^{t A} T e^{-t A}\right\| \leq C(1+|t|)^{\alpha},
$$

for all $t \in \mathbb{R}$ and for some constant $C>0$. Then the following conditions are equivalent:

(a) $T X_{A}(F) \subseteq X_{A}(F)$ for every closed set $F \subset \mathbb{C}$.

(b) $\Delta_{A}^{[\alpha]+1}(T)=0$.

In particular, if $0 \leq \alpha<1$, then $A T=T A$ if and only if $T X_{A}(F) \subseteq X_{A}(F)$ for every closed set $F \subset \mathbb{C}$.

Proof. (a) $\Rightarrow$ (b) We have

$$
\left\|e^{t \Delta_{A}}(T)\right\|=\left\|e^{t A} T e^{-t A}\right\| \leq C(1+|t|)^{\alpha}, \quad \forall t \in \mathbb{R} .
$$


Since $A$ is decomposable, $\Delta_{A}$ has SVEP [12, Proposition 3.4.6] and therefore,

$$
r_{\Delta_{A}}(T)=\varlimsup_{n \rightarrow \infty}\left\|\Delta_{A}^{n}(T)\right\|^{\frac{1}{n}} .
$$

On the other hand, $T X_{A}(F) \subseteq X_{A}(F)$ for every closed set $F \subset \mathbb{C}$ if and only if

$$
\lim _{n \rightarrow \infty}\left\|\Delta_{A}^{n}(T)\right\|^{\frac{1}{n}}=0
$$

12. Corollary 3.4.5]. Consequently, $r_{\Delta_{A}}(T)=0$ and so $\sigma_{\Delta_{A}}(T)=\{0\}$. By Corollary $2.8, \Delta_{A}^{[\alpha]+1}(T)=0$.

(b) $\Rightarrow$ (a) If $\Delta_{A}^{[\alpha]+1}(T)=0$, then $\lim _{n \rightarrow \infty}\left\|\Delta_{A}^{n}(T)\right\|^{\frac{1}{n}}=0$. By [12, Corollary 3.4.5], $T X_{A}(F) \subseteq X_{A}(F)$ for every closed set $F \subset \mathbb{C}$.

Remark 3.6. The proof of $(\mathrm{b}) \Rightarrow(\mathrm{a})$ in Proposition 3.5, can be simplified as follows: It suffices to show that $\sigma_{A}(T x) \subseteq \sigma_{A}(x)$ for every $x \in X$. If $x \in X$ and $\lambda \in \rho_{A}(x)$, then there is a neighborhood $U_{\lambda}$ of $\lambda$ with $u(z)$ analytic on $U_{\lambda}$ having values in $X$ such that $(z I-A) u(z)=x$ for all $z \in U_{\lambda}$. Using this, it is easy to check that the function

$$
v(z):=T u(z)-\Delta_{A}(T) u^{\prime}(z)+\ldots+(-1)^{k} \Delta^{k}(T) u^{(k)}(z) \quad(k=[\alpha])
$$

satisfies the equation $(z I-A) v(z)=T x$ for all $z \in U_{\lambda}$. This shows that $\lambda \in$ $\rho_{A}(T x)$.

Proposition 3.7. Assume that the operators $A, T$ in $B(X)$ satisfy the following conditions:

(i) $A$ is decomposable and $\sigma(A) \subset \mathbb{C} \backslash \mathbb{R}_{-}$, where $\mathbb{R}_{-}=\{t \in \mathbb{R}: t \leq 0\}$.

(ii) $\left\|A^{n} T A^{-n}\right\| \leq C(1+|n|)^{\alpha}(0 \leq \alpha<1)$ for all $n \in \mathbb{Z}$ and for some constant $C>0$.

Then $A T=T A$ if and only if $T X_{A}(F) \subseteq X_{A}(F)$ for every closed set $F \subset \mathbb{C}$.

Proof. It is trivial to show that if $A T=T A$, then $\sigma_{A}(T x) \subseteq \sigma_{A}(x)$ for every $x \in X$. It follows that $T X_{A}(F) \subseteq X_{A}(F)$ for every closed set $F \subset \mathbb{C}$. Now, assume that $T X_{A}(F) \subseteq X_{A}(F)$ for every closed set $F \subset \mathbb{C}$. For $z \in \mathbb{C} \backslash \mathbb{R}_{-}$, let $f(z):=\log z$ is the principal branch of the logarithm. Then $A=e^{B}$, where $B=\log A:=f(A)$. Notice that $B$ is decomposable [12, Theorem 3.3.6]. Moreover, if $t \in \mathbb{R}$, then as $t=n+r$, where $n \in \mathbb{Z},|r|<1$, and $|n| \leq|t|$, we can write

$$
\begin{aligned}
\left\|e^{t B} T e^{-t B}\right\| & =\left\|e^{r B} e^{n B} T e^{-n B} e^{-r B}\right\| \\
& \leq C\left\|e^{B}\right\|\left\|e^{-B}\right\|(1+|n|)^{\alpha} \\
& \leq C\left\|e^{B}\right\|\left\|e^{-B}\right\|(1+|t|)^{\alpha} .
\end{aligned}
$$

Since

$$
X_{B}(F)=X_{A}\left(f^{-1}(F)\right),
$$

for every closed set $F \subset \mathbb{C}[12$, Theorem 3.3.6], we get

$$
T X_{B}(F)=T X_{A}\left(f ^ { - 1 } ( F ) \subseteq X _ { A } \left(f^{-1}(F)=X_{B}(F) .\right.\right.
$$

By Proposition 3.6, $B T=T B$ which implies $e^{B} T=T e^{B}$. Hence $A T=T A$. 


\section{REFERENCES}

[1] E. Albrecht, Funktionalkalküle in mehreren veränderlichen für stetige lineare operatoren auf Banachräumen, Manuscripta Math. 14(1974), 1-40.

[2] E. Albrecht, On some classes of generalized spectral operators, Arch. Math. (Basel), 30(1978), 297-303.

[3] F.F. Bonsall and C. Duncan, Complete Normed Algebras, Springer-Verlag, New York, 1973.

[4] K.N. Boyadzhiev, Sinclair type inequalities for the local spectral radius and related topics, Israel J. Math. 57(1987), 272-284.

[5] I. Colojoară and C. Foiaş, Theory of Generalized Spectral Operators, Gordon and Breach, New York, 1968.

[6] J.A. Deddens, Another description of nest algebras in Hilbert spaces operators, Lecture Notes in Math. 693(1978), 77-86.

[7] I. Gelfand, Zur theorie der charactere der abelschen topologischen gruppen, Mat. Sb. 9(1941), 49-50.

[8] I. Gelfand, D. Raikov, and G. Shilov, Commutative Normed Rings, Chelsea Publ. Company, New York, 1964.

[9] E. Hille, On the theory of characters of groups and semi-groups in normed vector rings, Proc. Nat. Acad. Sci. USA. 30(1944), 58-60.

[10] V.P. Gurarii, Harmonic analysis in spaces with weight, Trans. Moscow Math. Soc. 35(1979), 21-75.

[11] R. Larsen, Banach Algebras, Marcel Dekker, New York, 1973.

[12] K.B. Laursen and M.M. Neumann, An Introduction to Local Spectral Theory, Oxford, Clarendon Press, 2000.

[13] Yu.I. Lyubich, Introduction to the Theory of Banach Representation of Groups, Oper. Theory Adv. Appl. vol.30, Birkhäuser, 1988.

[14] H.S. Mustafayev, Dissipative operators on Banach spaces, J. Funct. Anal. 248(2007), 428-447.

[15] H.S. Mustafayev, Growth conditions for conjugate orbits of operators on Banach spaces, J. Oper. Theory, 74(2015), 281-306.

[16] B. Sz.-Nagy and C. Foias, Harmonic Analysis of Operators on Hilbert Space (in Russian), Mir, Moscow, 1970.

[17] J.M.A.M. van Neerven, The Asymptotic Behaviour of Semigroups of Linear Operators, Oper. Theory Adv. Appl. vol.88, Birkhäuser, Basel, 1996.

[18] P.G. Roth, Bounded orbits of conjugation, analytic theory, Indiana Univ. Math. J. 32(1983), 491-509.

[19] J.P. Williams, On a boundedness condition for operators with a singleton spectrum, Proc. Amer. Math. Soc. 78(1980), 30-32.

[20] M. Zarrabi, Spectral synthesis and applications to $C_{0}$-groups, J. Austral. Math. Soc. Ser. A60(1996), 128-142.

[21] J. Zemanek, On the Gelfand-Hille theorems, In Functional Analysis and Operator Theory, Banach Center Publications, (ed. J. Zemanek), 30(1994), 369-385.

Van Yuzuncu Yil University, Faculty of Science, Department of Mathematics, VANTURKEY

E-mail address: hsmustafayev@yahoo.com 